Décadrages Décadrages

cınéma, à travers champs Cinéma, à travers champs

$14 \mid 2009$

Cinéma et migration

\title{
A propos de Walpurgis, de Frédéric Choffat
}

\section{Alain Freudiger}

\section{OpenEdition}

\section{Journals}

Édition électronique

URL : http://journals.openedition.org/decadrages/341

DOI : $10.4000 /$ decadrages.341

ISSN : 2297-5977

\section{Éditeur}

Association Décadrages

\section{Édition imprimée}

Date de publication : 1 avril 2009

Pagination : 111-117

ISBN : 978-2-9700582-9-8

ISSN : 2235-7823

Référence électronique

Alain Freudiger, «A propos de Walpurgis, de Frédéric Choffat », Décadrages [En ligne], 14 | 2009, mis en ligne le 10 avril 2010, consulté le 27 avril 2019. URL : http://journals.openedition.org/decadrages/341 ; DOI : 10.4000/decadrages.341 


\section{A propos de Walpurgis, de Frédéric Choffat}

\author{
par Alain Freudiger
}

Adapter le texte de Karl Kraus Troisième nuit de Walpurgis, écrit en 1933 dans les premiers mois qui suivirent la prise de pouvoir des Nazis en Allemagne ${ }^{1}$, est une tentative périlleuse autant que courageuse. La langue de Kraus, en effet, est d'une richesse et d'une finesse remarquables, complexe malgré sa grande clarté, tant elle est parcourue d'ironie, d'emportements, de brisures et surtout fragmentée en plusieurs voix au fil des nombreuses citations qui nourrissent le texte. Comment, dès lors, en rendre compte?

Frédéric Choffat a choisi de se baser sur l'adaptation scénique qu'en avait proposé José Lillo, au Théâtre Saint-Gervais de Genève, en mai 20072. S'il est parfois difficile de démêler ce qui, dans le film, relève du texte de Kraus, de la magnifique traduction de Pierre Deshusses, de la version théâtrale de José Lillo, de l'adaptation par Frédéric Choffat et Julie Gilbert et de la réalisation de Choffat, l'ensemble forme une toile épaisse où s'enchevêtre un nombre considérable de niveaux textuels. Choffat a en effet choisi de multiplier, sinon les lieux de parole - le tournage a entièrement eu lieu dans un champ gelé à l'orée d'un bois - du moins les modes d'inscription des voix: celle, face à la caméra, du comédien José Lillo (le même qui a mis en scène la pièce à Saint-Gervais); la voix over; les inscriptions dans l'image (intertitres, sous-titres...) et même le commentaire musical, par le biais de la composition crépusculaire du duo électroacoustique Kristoff K. Roll. Dans ce foisonnement de couches textuelles, les niveaux d'énonciation renvoient d'une part à des niveaux déjà inclus dans le texte de Kraus lui-même, que ce soit dans son édition française ou dans son adaptation scénique, et d'autre part à des niveaux inhérents au médium cinématographique. Pour la première catégorie, on peut distinguer le texte écrit par Kraus, les citations qu'il y ajoute, la traduction par Pierre Deshusses, les indications métatextuelles et l'interprétation de José Lillo inspirée, dans le choix des extraits et le ton employé, par son spectacle genevois. Mais au final, toutes ces couches textuelles sont mises en voix au sein du film par Lillo seul. Quant aux
1 Karl Kraus, satiriste et polémiste viennois, commence Troisième nuit de Walpurgis (ainsi nommé en référence au "Second Faust" de Goethe) en mai 1933, et le termine en octobre 1933. Ce texte, réquisitoire implacable contre le nazisme et long cri d'effroi devant ses horreurs, ne sera pas publié du vivant de Kraus, et ne paraîtra dans son intégralité en Allemagne qu'en 1952. Kraus en livre toutefois de larges extraits dans le cahier de janvier-février 1934 de Die Fackel, le "contre-journal " qu'il a fondé en 1899. Karl Kraus, Troisième nuit de Walpurgis, traduit de l'allemand par Pierre Deshusses, Editions Agone, Marseille, 2005.

2 Cette mise en scène fut d'ailleurs à l'origine le projet du film lui-même, comme l'indique le dossier de presse: "Le projet Walpurgis est né à la suite d'une mise en scène de José Lillo présentée à St-Gervais en mai 2007 sur un texte de Karl Kraus", et le film porte la mention "Sur une proposition de José Lillo". Ce dernier incarne par ailleurs le "personnage" central du film. 
3 Parlant non de ce texte précisément mais de la prose de Kraus en général, Wilhem écrit: "En somme, l'ironiste (qui n'oublie jamais la parodie ou, si l'on préfère, une certaine mascarade) n'écrit pas en filant, en tirant ce qu'il énonce: il fait des phrases; il tente de réussir celle-ci, puis celle-là, et celle-là encore, non pour composer une suite, mais pour ouvrir deux voies, pour les donner à choisir comme elles s'écartent ou comme elles s'élargissent, le temps qu'elles restent plus ou moins ouvertes, et donc fourchées. En sorte que, d'une phrase à l'autre, d'une bifurcation à l'autre, l'auteur n'avance pas, il ne progresse pas en tenant un discours général. II élève plutôt un mur de pierres." Daniel Wilhem, "Le mur de Vienne", in Bibliomanie, Ligne et manifeste, juin 2006, p. 33. Une première version du texte, un peu différente, est parue sous le même titre dans la revue Furor, $n^{\circ} 6,1982$. Certes, Troisième nuit de Walpurgis n'est pas n'importe quel texte de Kraus. Ce dernier a en effet peiné à trouver des mots aptes à rendre compte du nazisme. On retrouve cependant des procédés courants chez cet auteur, tels que l'ironie et la bifurcation.

4 "On peut encore trouver des formulaires servant à attester qu'il n'est rien arrivé à quelqu'un qui vient d'être torturé; ils sont à la disposition de tous ceux qui parviennent à sortir sains et saufs d'une Maison brune." Kraus, op. cit., p. 230.

$\mathbf{5}$ Le film est d'ailleurs très bien rythmé: les tirades de Lillo formant le corps sont entrecoupées par des silences, des plans noirs, des plans sur la forêt ou l'intensification de la musique, avec des pauses plus ou moins longues. niveaux plus directement cinématographiques, on peut mentionner les intertextes (indications de contexte, etc.), les citations défilant en soustitres dans l'image, les mots ou phrases apparaissant en surimpression à l'écran (jusqu'à l'obstruer parfois), le jeu sur les écrans (écran dans l'écran, split-screen), les ponctuations (plans noirs, fondus) après une tirade ou un extrait, la musique électroacoustique, et bien sûr le niveau de l'image elle-même: José Lillo, filmé de face en gros plan et en noirblanc alternant avec les plans de paysage en couleur.

Ce matériau composite et comme empilé ne renvoie pas précisément à ce que Daniel Wilhem a appelé «le mur de Vienne»3, mais il érige tout de même une sorte de mur qui tente de faire résonner le texte de Kraus par des systèmes d'équivalences. Troisième nuit de Walpurgis, en effet, est une longue indignation, description, mise en faits, collage de citations, relation, confrontation de paroles et d'actes, à l'ironie terrible 4 . Loin d'un quelconque ressassement, on est plutôt dans la multiplicité des points d'ancrage, fragiles mais très précis, mesurés au plus près, comme un mécanisme de drainage qui ferait entendre clairement ce qui sort vicié de la bourbe des mensonges et des contradictions. Or, que fait Choffat dans son adaptation cinématographique? Il tisse et met en place un discours continu mais morcelé, procédant par coupes, marquant ainsi des équilibres tendus entre des niveaux expressifs. Il n'y a pas lieu ici d'entreprendre une analyse systématique de ces niveaux et de leur articulation pour en tirer une hypothétique grammaire, d'autant plus qu'à mon sens cette multiplicité a avant tout un intérêt rythmique $\mathbf{5}$ - elle ponctue, ouvre, ferme, tourne, souligne, commente, incruste, rendant en cela justice à son matériau au niveau formel. Je note, par exemple, que les citations faites par Kraus (lorsqu'il cite explicitement, non quand

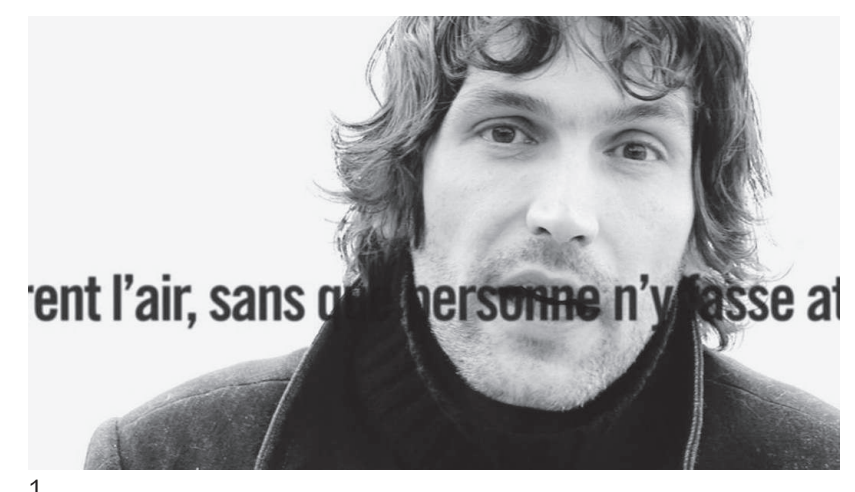

1

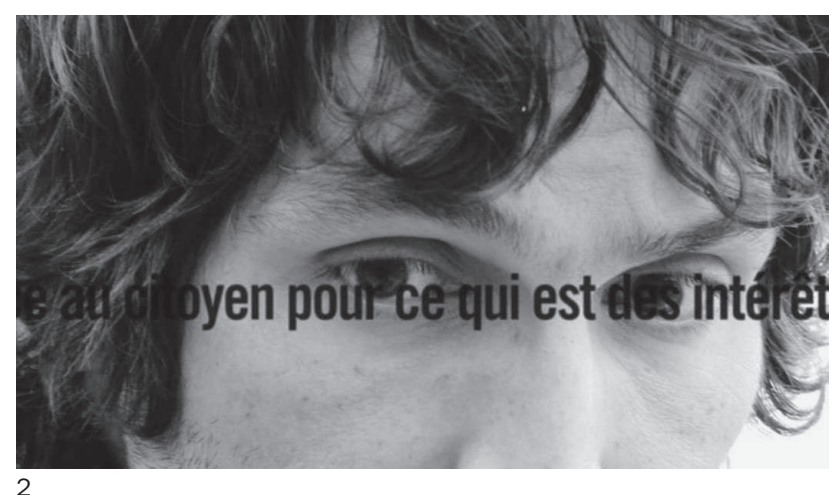


il les intègre à sa prose) et dites par Lillo sont systématiquement rendues sensibles à l'écran. Le plus fréquemment défilent en surimpression des extraits de Faust ou de Wagner. Selon les séquences, l'inscription en surimpression se positionne différemment sur l'écran par rapport au comédien, et ferme parfois d'autres sources d'expression, comme dans cette citation de Macbeth vers la fin du film ${ }^{6}$ qui défile sur la bouche de Lillo jusqu’à le bâillonner (fig. 1), ou celle de Wagner qui «bouche» les yeux de l'interprète, créant ainsi un effet de redondance ironique:

"Si le patriotisme donne des dons de voyance au citoyen pour ce qui est des intérêts de l'Etat, il le rend encore totalement aveugle pour ce qui est des intérêts de l'humanité en général...» (fig. 2)

Cette superposition implique que les niveaux de parole ne peuvent s'actualiser en même temps et que certains doivent être "suspendus" pour laisser brièvement plus de place à l'un ou pour construire une ombre portée sur un autre. Quoi qu'il en soit cette construction textuelle, claire chez Kraus (qui cite ses sources), l'est aussi chez Choffat - et même doublement, puisque outre l'inscription, José Lillo mentionne à chaque fois l'auteur de la citation. Cette redondance maintient un équilibre entre des temps, des lieux et des niveaux d'expression différents.

En revanche, l'équilibre est brisé avec certaines autres interventions graphiques: ainsi, les chiffres digitaux (fig. 3) qui créent un rapport entre 1933 et 2008 établissent une relation trop directe et injustifiée, rompent maladroitement la tension entre les temps et les lieux, et vont jusqu'à mettre à mal l'équilibre fragile des niveaux d'expression, puisqu'ils s'étalent sur tout l'écran, sur fond noir. Ils font alors disparaître Lillo, le paysage et la voix au profit d'un "compte à rebours» sursignifié. D’autres effets de soulignement s'avèrent déséquilibrés : la répétition de la phrase "si on se bouche les oreilles on n'entend plus aucun râle", redoublée par
6 "Pauvre empire qui de lui-même s'effraie. II ne peut plus être appelé notre mère patrie, seulement notre tombeau: où seul sourit qui ne sait rien; où les cris et les soupirs déchirent l'air sans que personne n'y fasse attention. Personne ne demande: pour qui? quand sonne le glas? La vie des braves flétrit plus vite que le bouquet sur leur chapeau et meurt avant même de tomber malade. "William Shakespeare, Macbeth, cité par Karl Kraus, op. cit., p. 439.

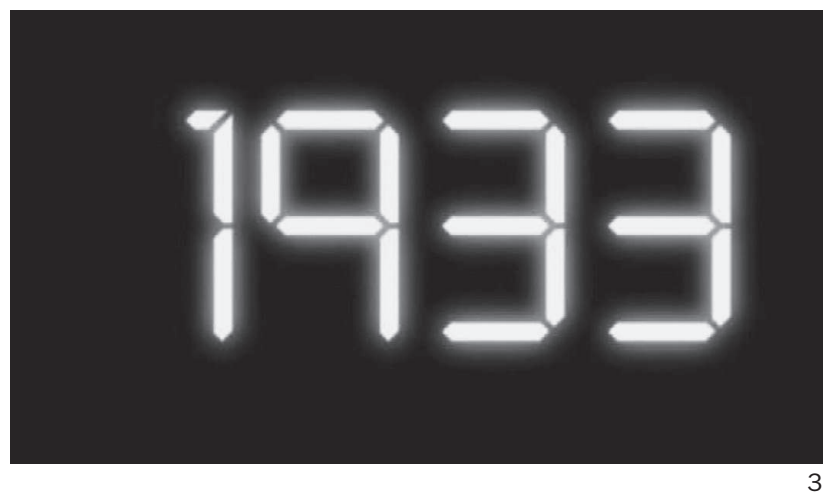


7 Karl Kraus, op. cit, p. 295.

8 Sur les influences du nazisme sur la langue allemande, on lira avec profit le livre de Victor Klemperer, LTI, la langue du Troisième Reich. Carnets d'un philologue, Albin Michel, coll. Bibliothèque Idées, Paris, [1947] 1996, 375 p. son inscription à l'écran et encore soulignée par l'interruption de la musique sinon omniprésente, aplatit l'expression, là où Kraus ne manque jamais de relief. Mais ces effets peuvent aussi fonctionner plus judicieusement, par exemple quand les lettres incrustées à l'écran tombent une à une jusqu'à occuper toute la place et à faire disparaître Lillo, au moment de son discours sur les sigles (fig. 4-5):

"La nomenclature se voit adaptée au désir pressant d'économiser du temps et de gagner de l'espace. Cet enrichissement par raccourcissement, qui nous a gratifiés d'abats lexicaux aussi appétissants que Hapag et Wipag, Afeb et Gesiba, Kadewe et Gakawe et autres formules magiques, est l'origine en vertu de quoi sont arrivés les Osaf et les Gausaf; on ne sait plus ce qui est de plus mauvais augure : lorsque la Gestapo ou l'Uschla intervient, lorsque la NSBO se rebiffe ou le HHV se soumet [...]. Mais depuis qu'il y a la SS et la SA, il ne nous reste plus qu'à lancer un SOS aux USA. » 7

Avec ces lettres tombant par grappes, l'emprise d'une langue «recadrée » 8 est oppressante, finissant par enfermer Lillo derrière un grillage. Au-delà ou à l'inverse, certaines paroles de Lillo sont prononcées sur un écran noir, ce qui, en disloquant les lieux de parole (la voix est-elle encore in ou off?) donne une sorte d'ouverture latérale, issue ou point d'entrée, qui déjoue la frontalité du dispositif - par le verbal et contre l'image, en quelque sorte. Quant au jeu sur les split-screens, il ne fonctionne pas selon une véritable systématique, et si les effets sont ici ou là raisonnablement convaincants, notamment en recadrant celui qui se met en scène (citations de Goebbels ou de Hitler), les autres font un peu "procédé». Je retiens juste un plan plus particulièrement expressif: un splitscreen de quatre images figées de Lillo, lorsqu'il cite les propos nazis sur la «femme allemande», où finalement, au ralenti, sur l'image en bas à
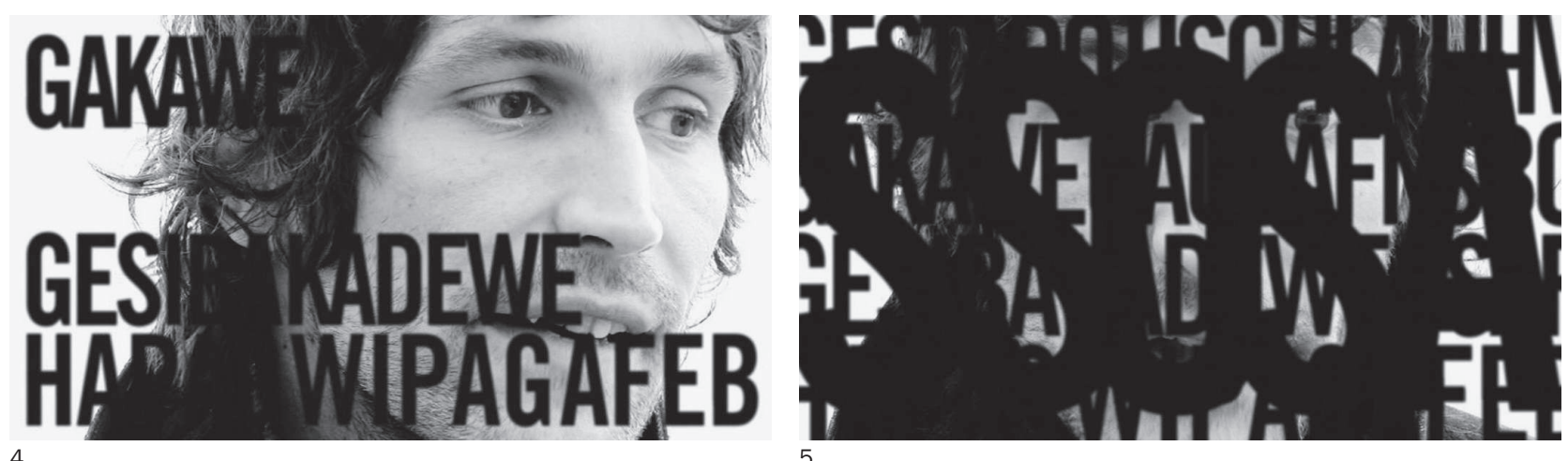


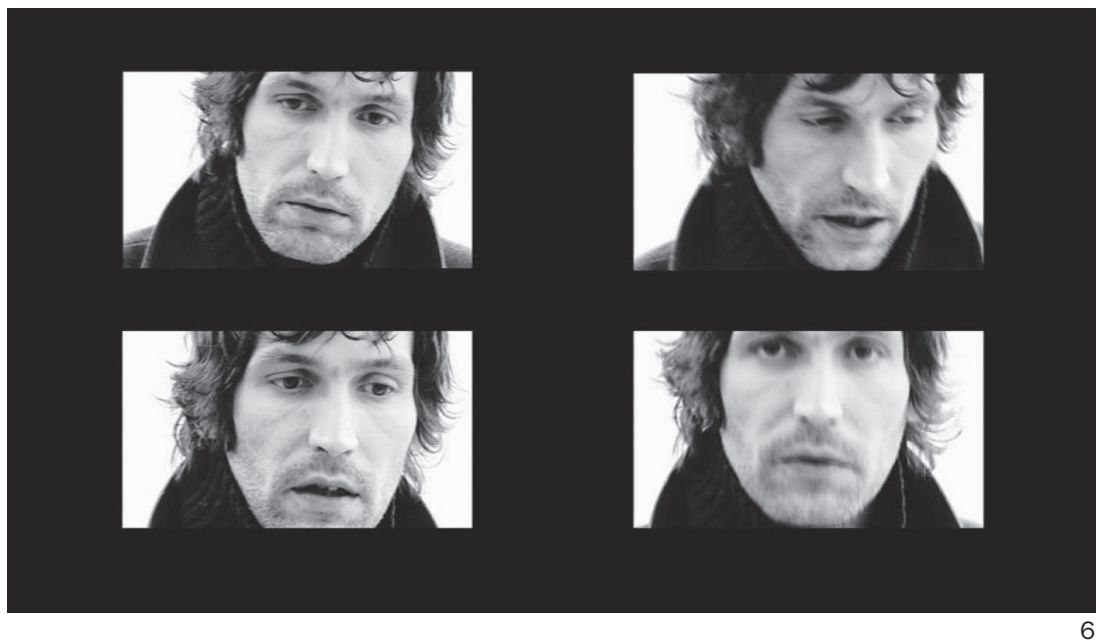

droite, Lillo relève la tête et regarde frontalement la caméra, alors qu'on entend sa voix over dire : «... il existe encore des jeunes filles allemandes qui n'ont pas honte de se montrer en public avec des Juifs.» (fig. 6).

Si les inscriptions graphiques ne sont pas toujours un choix convaincant, l'image, elle, l'est. Il y a principalement deux types de plans: ceux sur José Lillo, et ceux sur le paysage, qui fonctionnent comme ponctuations ou comme respirations. Les plans sont parfois nets, parfois légèrement flous, notamment ceux sur la forêt qui jouent sur la focale et sont régulièrement tremblés; ils recèlent aussi une ambiguité entre la couleur et le noir et blanc, de par leurs tons pâles et hivernaux. Cette incertitude souligne la fragilité du lieu, non seulement d'où l'on peut parler, mais dont on parle. Mais ce sont les plans sur José Lillo qui sont majoritaires. Toujours en noir et blanc et surexposés, ils évoluent en partie au cours du film. L'exposition du «personnage» est à ce titre exemplaire: le film s'ouvre d'abord sur des plans de forêt qui sont suivis par celui du visage de José Lillo. Alors que la caméra essaie de faire le point sur lui, il respire, dévisage la caméra et dit: "Je n’ai aucune idée sur Hitler». La phrase est répétée en voix over, mais cette fois en allemand, puis vient un plan noir. La "disparition» de José Lillo à la fin du film en revanche se fait via un fondu au blanc - le film effectue une sorte de trajet du sombre vers le clair. Par ailleurs José Lillo a aussi une valeur "plastique», malheureusement un peu gâtée par le choix de la surexposition qui lisse son visage: tout en regard perçant et en bouche dentue, avec des cheveux légèrement désordonnés, il est 
9 II n'est pas sans intérêt de savoir que Kraus lui-même était un grand lecteur public.

10 Ce silence est peut-être le renversement de celui qui frappa Kraus lui-même: "A l'obligation d'écrire, Kraus a substitué, pendant les premiers mois de l'année 1933, celle de prendre la mesure de la catastrophe. Comme un acteur de théâtre qui fait de son silence un soutien de la réponse à venir.

Je reste cois;

et ne dis pas pourquoi.

Et il y a du silence, alors que la terre craquait.

Aucune parole qui touchait; [...] ensuite c'était indifférent.

La parole s'endormait lorsque ce monde s'éveillait,

fait-il paraître dans le bref numéro qui précède Troisième nuit de Walpurgis, dont le texte était destiné au départ à faire tout un numéro de Die Fackel. ", Pierre Deshusses, "En traduisant dritte Walpurgisnacht", in Karl Kraus, op. cit., p. 8.

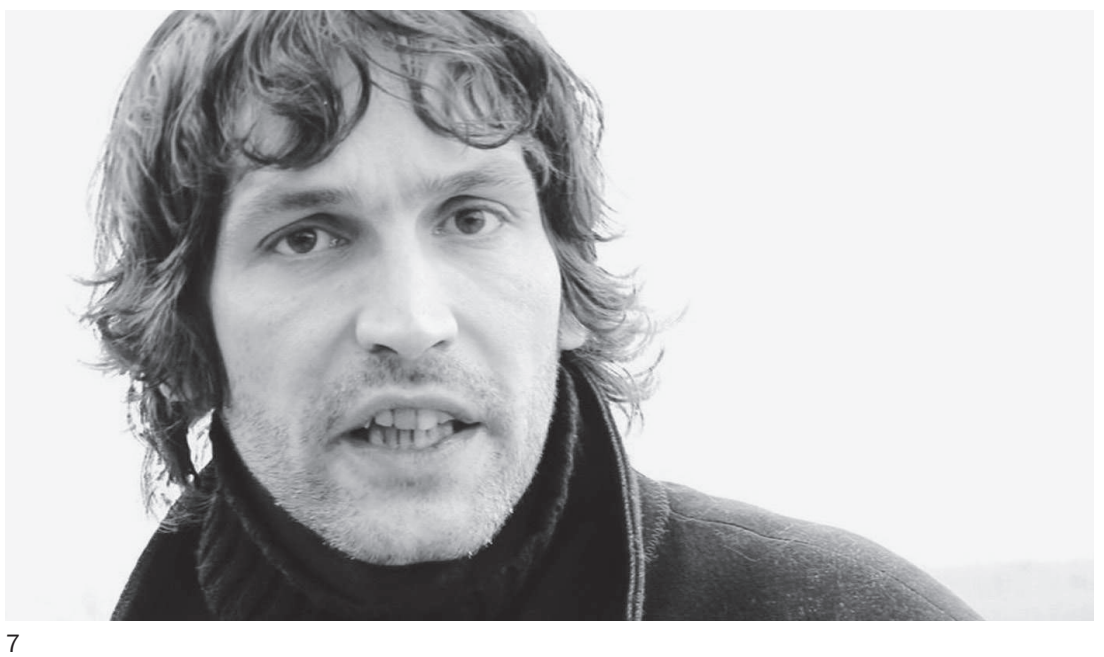

à la fois intemporel et extrêmement incarné, charnel (fig. 7), ce qui est encore souligné par sa diction.

Je qualifierais celle-ci de bouchue, mâchée, avec un léger accent, comme si Lillo avait quelque chose dans la bouche, ce qui donne à sa voix un volume non seulement sonore mais matériel. Il ne s'agit pas du tout d'un flow de slameur, mais plutôt d'un balisage, d'une mise en place très variée: hausses de ton, ralentissements, accentuation d'une syllabe pour signifier ici ou là un décalage, une ironie ou une citation, ou encore une mise à distance de certains mots («national»...), jusqu’à user d'une voix suraiguë et un peu ridicule lorsqu'il cite Gottfried Benn, hoquetant le mot "peuple». Une palette désarçonnante, ni incarnation ni distanciation, un travail remarquable, qui rend justice au texte de Kraus ${ }^{9}$. Il est dommage que sa parole soit souvent brouillée ou atténuée par la musique, dont l'omniprésence nuit au texte proféré. Car le texte de Kraus, me semble-t-il, est si dense qu'il creuse une sorte de vide autour de lui; un vide, non entre les mots ou entre les lignes, mais un vide devant et derrière lui, avant et après lui. Ce vide n'a rien d'un vide absolu, immense ou infini; c'est un vide localisé, temporaire et presque fragile, un vide qui autorise, non un écho, à peine une résonance, mais une prise des mots. Face à ce texte, on est comme abasourdi - un instant; il faut le poser, le reposer, et c'est alors que se fait, bref et précaire, un vide, un vide qui est un espace et qui est aussi un silence $\mathbf{1 0}$ : un vide qui permet à cette voix de prendre précisément sa dimension - juste avant d'être menacée et engloutie par des échos, des fureurs, des bruits, des nuits et des brasiers. 
Or il me semble que Choffat, en plaçant José Lillo à l'orée de cette forêt sur cette terre gelée $\mathbf{1 1}$, a perçu l'importance de ce vide; que sa voix, que sa parole sont non pas portées, ni même répercutées par le lieu, mais pas non plus absorbées; qu'elles tombent, raides et précises, et qu'elles ont la place de le faire, qu'un lieu vide leur répond - vide, mais pas engloutissant. Et que ce lieu vide est fragile, menacé déjà par la forêt et son ombre noire, par le gel qui lisse la prairie, mais que ce lieu existe, qu'il y a une place pour penser et pour dire, une place sans écho. En cela, oui, le film de Choffat est bien un "essai cinématographique", même s'il aurait gagné à rester sur son dispositif de base (articulation de plans sur José Lillo et de plans sur le paysage) et à éviter des effets qui trop souvent atténuent la force du texte.

11 "Avec le comédien José Lillo, les essais se sont succédés, d'entrepôts en voies ferrées, de parkings en supermarchés, jusqu'à ce que finalement je décide de tourner au milieu d'un champ en plein hiver, à l'orée énigmatique d'un bois. Parallèlement à l'enregistrement du texte, j'ai cherché dans ce paysage de campagne un contrepoint à cette parole drue. Et jour après jour, j'ai filmé l'orée comme un deuxième personnage du film. "Frédéric Choffat, dans le dossier de presse du film. Je ne dirais pas pour ma part qu'il s'agit d'un personnage, cependant. II ne tient pas de rôle ou de place: il permet le jeu et la disposition.

Walpurgis (Suisse, 2008, $60 \mathrm{~min}$.)

Réalisation: Frédéric Choffat. Production: Frédéric Choffat / Les films EEilSud. Scénario : Frédéric Choffat, Julie Gilbert, d'après Karl Kraus. Image: Frédéric Choffat. Montage: Frédéric Choffat. Son: Gilles Abravanel. Musique: Kristoff K. Roll. Interprétation: José Lillo 